\title{
The influence of multi objective programming on Aerobics Teaching
}

\author{
Yali $X u^{1}$, Kaihua $\mathrm{Xu}^{2}$ \\ ${ }^{1}$ School of Physical Education, Jiangxi University Of Traditional Chinese Medicine, Nanchang, 330004, \\ China \\ ${ }^{2}$ Police Training Management Department, Jiangxi People's Police College, Nanchang, 330103, China
}

Keywords: Multi-objective programming, Aerobics, Teaching, Impact.

\begin{abstract}
This paper explore application method of multi-objective programming and discussed its effect in aerobics teaching, through the analysis of the summary of multi-objective programming, studies the principles that need to follow in the multi-objective programming of aerobics teaching.
\end{abstract}

\section{Introduction}

Multi-objective programming, also known as multi-objective optimization, is the method of optimizing more than one objective function in a given region. In the process of teaching management, decision making and planning daily, often there will be many different solutions and to achieve the goal, decision makers need more than optimal through the contradictory relationship between the target and a target analysis to find the solution of the problem, and the multi-objective planning is to help decision makers the multi-objective problem into a single objective analysis, so as to achieve the purpose of seeking common optimal solution.

The traditional aerobics teaching pays too much attention to the training of students' skills and skills, and ignores the influence and effect of Aerobics on students' lifelong development. With the development of the reform of physical education, the aerobics teaching in the new period has more diversified and diversified goals. According to the developmental needs of students and the teaching of aerobics, the aerobics teaching needs combined with multi-objective programming, to find out the teaching objectives of the "optimal solution", comprehensively enhance the quality of teaching of Aerobics .

\section{Summary of multi-objective Programming}

In real life, the decision is often accompanied by multiple objectives coordination and balance, many target itself is contradictory and conflicts, the problem solving process become more complicated and difficult choices. For example, shopping in the consumer, not only consider the practical value, brand, price and packing etc. certain items, but also consider whether it can meet their psychological goals, expectations and opinions of others to buy the achievement of objectives through various purchasing decisions.

Multi objective programming is not only the result of modern management science, but also a systematic analysis method. Its goals are often contradictory, non public and inconsistent. Multi objective programming is to study the optimization problem that can not be compared, and adopt the different methods of solution, stratification, or exclusion to achieve the optimal solution, and help the decision-maker to find the optimal target. 


\section{The principles to be followed in the application of multi objective programming in Aerobics Teaching}

\section{Simplicity principles}

Aerobics is a kind of sport which combines bodybuilding and body-building. It is popular with young people because of its aesthetic, physical and lively characteristics. In the actual teaching of aerobics, often faced with multiple choices such as teaching objectives, improve physical function, exercise habits, good shaping, enhance students' willpower and improve students' sense of teamwork and sense of collective honor etc.. The concise principle of multi-objective programming is based on the analysis of each target and the decision-making, as far as possible to reduce the target number, such as the merger of similar targets by simplifying objectives, excluding affiliated methods and through the goal, will only reach the standard reduction targets as constraint conditions, the method to measure the same sum, the average or comprehensive function, achieve comprehensive index instead of single index objective.

\section{Choosing principle}

In the solution to the problem, in order of priority and to reach the goal of real resources and other conditions, can determine the order of the relationship between multiple objectives, the importance of [1] will target to sort. For example, the multi-objective programming of aerobics teaching, and teaching students to solve practical problems facing the needs of the target can be sorted, such as enhancing the cultivation of willpower $>$ to develop exercise habits $>$ to improve the sense of cooperation >to enhance physical fitness. The goals are sorted according to the importance, and the important coefficients of each objective are stipulated so that the choice of the optimal plan can be carried out according to the importance of the objective.

\section{Coordination principle}

Multi-objective programming often faces the problem of multiple objectives, namely, the goal of achieving a goal needs to be eliminated or violated at the expense of other goals. When facing the choice of multiple goals, we should obey the principle of coordination. Under the guidance of the general objective, we should consider the relationship among the various objectives and coordinate the conditions of each goal. For example, in the actual teaching of aerobics, guidance to promote students' overall goal of Aerobics knowledge, realize students' cooperative consciousness and the spirit of collectivism, the "beauty" of cognition and social adaptability of the target, for each target for consideration..

\section{The concrete method of multi objective programming in Aerobics Teaching}

\section{Dissolve more into less}

Dissolve more into less is actually on the process of aerobics teaching target do subtraction, by using simple principle of multi project planning, multiple targets into only two or a target, in order to find out the optimal solution of the problem "'. Among them, there are many methods which can be divided into comprehensive scoring method, square sum method and constraint method. In the planning of aerobics teaching, the application of comprehensive scoring method is mainly carried out .

First of all, using the method of decision makers need to determine the evaluation target according to the actual situation of aerobics teaching, i.e. what index evaluation in aerobics teaching, can be divided into physical quality, mental outlook, social skills and team evaluation index. [2] secondly, decision makers need to combining qualitative index and quantitative index, according to the evaluation of the goal to develop a corresponding evaluation grade and score range, and then according to the index to develop evaluation criteria in aerobics teaching, can be divided into excellent, good, medium and poor four grades. Thereafter, the decision maker needs to make a scoring table containing three elements, namely, evaluation index, scoring and grading. Thirdly, the 
decision maker should select the numerical value according to the relevant data collected, and deal with the data and the corresponding evaluation. Finally, it is a comprehensive evaluation of the results of the object, and comprehensive use of the results.

\section{Hierarchical sequence method}

Hierarchical sequence can also be called priority method, that is, each goal is sorted or stratified according to priority, and solved according to the order of arrangement. In aerobics teaching, the primary goal of decision-makers to consider is often the physical quality of the students or to master the aerobics skills, but in the teaching reform of physical education requirements of the new era, the development of social demand and the principle of aerobics teaching target need of students, the teaching goal setting in the long term, the aerobics teaching runs through the growth of students. So, in the classification column on aerobics teaching, can achieve short-term goals and long-term development goals on students, the future training goal > skills, evaluation of social ability training > sports performance, the spirit and culture training $>$ and enhance body quality, the evaluation team cooperation ability > person ability, and ultimately achieve the goal of unity, the adapt to the development of students, make students benefit from lifelong aerobics teaching target .

\section{Analytic hierarchy process}

Analytic hierarchy process can also be called the analytic hierarchy process, the system is a combination of qualitative and quantitative multi objective induction method has strong practicability and application value for the complex problem solving contradiction, correlation. In aerobics teaching, the application of the AHP can be divided into the following steps: one is to establish the hierarchical structure model of the system, can be divided into the target layer, criterion layer and layer three layers, layer from top to bottom and then according to various factors, affect the physical and mental outlook such as aerobics the effect of teaching, teamwork ability and ability to adapt to society, construction of the comparison matrix using pairwise comparison method, until the lowest level. [3] two is based on each of the criteria for each factor to compare, such as physical quality indicators easier to test, and the spirit of next. Or team work ability is short, time is more easy to reach, and social adaptation ability next. Three, it is necessary to make a comprehensive analysis of the two levels of judgment, and to select the optimal scheme.

\section{The influence of multi objective programming on Aerobics Teaching}

\section{Providing a more scientific direction for Aerobics Teaching}

The correct target is the premise and foundation to achieve the teaching tasks, the evaluation method is scientific and systematic by multi-objective programming, aerobics teaching activity indicates the direction of development, the important matters needing attention in the teaching activities and the optimal solution". In the traditional aerobics teaching, because of the lack of scientific guidance, teaching, and students always needs to PE teaching reform and the direction of social development combined, resulting in actual aerobics teaching work, too much attention to the students' skills training, ignoring the "wisdom" "Virtue" "beauty" teaching.

To solve the multi-objective programming, teaching can make up for the loopholes in the work and the vacancy, much less, hierarchical sequence analysis method and AHP method, teachers can clearly understand between the teaching objectives of the order of priority, timely feedback and solve the teaching problems. For example, in aerobics teaching goal setting, students have mastered the basic music, physical exercise skills of aerobics, so after weighing various factors, the current aerobics teaching target can develop to the broader, deeper and more long-term direction. When the new goal of progressiveness and socialite is set up, the teacher can carry out the design of the specific teaching plan according to the guidance of the target, and make sure that the aerobics teaching is going on the right way. 


\section{Ensuring aerobics teaching more suited to students' future development needs}

Teaching objectives should be set with the long-term development needs of students to meet the growth needs of students. In the traditional aerobics teaching target design, too often pay too much attention to the short-term achievements of students, ignoring the long-term education and training of students. In the multi-objective planning strategy, the teaching goal is not confined to a certain level and in the field, in the premise of having multiple alternatives, the optimal objective discuss the creation of the aerobics teaching work to provide a more diverse and diversified choices, in combing the various goals of contradictions and relations, decision able to multi objectives and balanced consideration, and more easily found point to the teaching goal, make considerations for the future development of students

The traditional aerobics teaching will often take a single target, such as a master in some aerobics skills, get a victory, but not from the overall and long-term aspects of systematic consideration of the development of students, the multi-objective planning method is to solve the problem. For example, in the multi objective planning of aerobics teaching, the decision maker needs to sort the priorities among the goals. In the sports teaching reform under the guidance of the decision makers will be able to see the students psychological quality, team awareness and social adaptation ability is very important for the future development, and as an important goal in the planning of the first, the other goals in the overall goal of the guidance, to develop to the long-term culture and social development.

\section{Improving the quality of Aerobics Teaching}

The level and quality of aerobics teaching are closely related to the choice of teaching objectives. The traditional aerobics teaching work is often not planning and scientific system of decision-making, implementation for each target will often take "hand in hand" and "both parties" strategy, teaching resources, but due to the limitation of time and space, often ended in failure, but don't waste the corresponding teaching resources for students healthy growth is also a kind of damage. Multi objective programming can make each target much less, structured, provided to the aerobics teaching plan and scientific reference, to enable students to truly feel the charm of the sports aerobics .

For example, in the aerobics teaching planning, decision makers often find the body to enhance the quality and psychological quality of students enhance the two factors of the contradiction, namely the improvement of bodily functions must be accompanied by more intensive training, and the training process will be easy to make students boredom and resentment. You need to apply to the multi-objective programming of balance principle and the hierarchical analysis method in aerobics teaching should not only guarantee a certain amount of exercise, the students' physical quality continue to improve, but also in the process of teaching to enhance the fun and team training methods, make students of physical and mental elements of balance, make the teaching task "appropriate". This is the teaching of aerobics will not lead to the imbalance of physical and psychological factors of students, can enhance the physical quality of the students, and cultivate students' psychological ability and strong willpower, to enhance the quality of Aerobics Teaching in all aspects .

\section{Conclusion}

Aerobics has characters of adaptability, times and targeting, it can upgrade students' sense of harmony, musicality, self-confidence, social skills and aesthetic ability. Multi objective planning should be combined with the characteristics of aerobics teaching, through scientific decision-making methods, and open system development, make teaching objectives more suitable for sports, society and students, and the teaching level and quality of Aerobics promotion, give full play to its positive influence on students growth in the future.

\section{Reference}

[1] Zhang Wen, Liu Yubin. A multi objective decision model for educational evaluation, Journal of Shandong Normal University (NATURAL SCIENCE EDITION), 2003, (01): 17-20. 
[2] Han Wenxiu. Application of multiple objective decision making in educational planning, Journal of Tianjin University, 1982, (03): 49-56.

[3] Liu Qinqin. Research on aesthetic experience and aesthetic function of Aerobics, Shandong Normal University, 2006. 